\title{
Antimicrobial susceptibility and serotype distribution of Streptococcus agalactiae rectovaginal colonising isolates from pregnant women at a tertiary hospital in Pretoria, South Africa: An observational descriptive study
}

\author{
M Said, ${ }^{1,2}$ BSc, MB BCh, DTM\&H, MMed (Microbiology), FCPath (SA) Micro; Y Dangor, ${ }^{1}$ MSc; \\ N Mbelle, ${ }^{1,2}$ MSc, MB BCh, DTM\&H, FCPath (SA) Micro; S A Madhi, ${ }^{3}$ MB BCh, MMed (Paediatrics), FCPaed (SA), PhD; \\ G Kwatra, ${ }^{3}$ MSc, PGDip (Pharmaceutical Regulatory Affairs), PhD; F Ismail, ${ }^{4}$ BSc, MB BCh, DTM\&H, MMed (Microbiology)

\begin{abstract}
${ }^{1}$ Department of Medical Microbiology, School of Medicine, Faculty of Health Sciences, University of Pretoria, South Africa
${ }^{2}$ National Health Laboratory Service, Tshwane Academic Division, Pretoria, South Africa

${ }^{3}$ Department ofScience and Technology/National Research Foundation: Vaccine Preventable Diseases, University of the Witwatersrand, Johannesburg; and Medical Research Council: Respiratory and Meningeal Pathogens Research Unit, University of the Witwatersrand, Johannesburg, South Africa ${ }^{4}$ TB Reference Laboratory, National Institute of Communicable Diseases, Johannesburg, South Africa
\end{abstract}

Corresponding author: M Said (msaid@mweb.co.za)

\begin{abstract}
Background. Streptococcus agalactiae or group B streptococcus (GBS) is a significant cause of neonatal sepsis. Intrapartum antibiotic prophylaxis is recommended for pregnant women identified to be rectovaginally colonised between 34 and 37 weeks' gestational age to decrease the risk of invasive disease in their newborns. An effective multivalent GBS vaccine may prevent a broader scope of GBS-associated diseases, such as GBS early-onset disease, GBS late-onset disease, spontaneous abortion, stillbirth and maternal bacteraemia. Serotype distribution of GBS isolates is essential to determine the efficacy of such a vaccine.

Objectives. To investigate serotype distribution and antimicrobial susceptibility patterns of GBS isolates cultured from rectovaginal specimens during pregnancy.

Methods. Sixty-nine archived maternal colonising isolates were tested against penicillin, erythromycin, clindamycin, vancomycin and levofloxacin. Minimum inhibitory concentration testing was performed using the ETEST method. Serotyping was performed by the latex agglutination method.

Results. The most common serotypes detected were Ia (54\%), III (20\%), V (16\%), II (6\%), IV (2\%) and Ib (1\%). All isolates were fully susceptible to penicillin, vancomycin and levofloxacin. Eight (11\%) and $50(56 \%)$ isolates showed intermediate resistance to erythromycin and clindamycin, respectively, and 1 isolate was resistant to erythromycin. The macrolide-lincosamide-streptogramin $B\left(\right.$ MLS $\left._{B}\right)$ phenomenon was noted in $3(4 \%)$ of the isolates.

Conclusions. GBS-colonising isolates remain susceptible to penicillin, which remains the drug of choice for intrapartum antibiotic prophylaxis and treatment of invasive disease in newborns. Macrolides should only be used if clinically indicated due to the high prevalence of intermediate resistance. A pentavalent GBS vaccine currently in phase I trials should provide coverage for $97 \%$ of the isolates identified in this study.
\end{abstract}

S Afr Med J 2020;110(9):869-871. https://doi.org/10.7196/SAMJ.2020.v110i9.14524

Streptococcus agalactiae or group B streptococcus (GBS) remains a significant cause of neonatal sepsis, responsible for early-onset disease (EOD) ( $<7$ days of age) and late-onset disease (LOD) (7 89 days of age). ${ }^{[1]}$ The incidence of EOD has declined significantly in countries where universal screening of pregnant women for GBS colonisation is undertaken between 34 and 37 weeks of gestational age and intrapartum antibiotic prophylaxis (IAP) during labour is provided to colonised women. ${ }^{[2]}$

Penicillin is the drug of choice for IAP and for the treatment of GBS-EOD and GBS-LOD. Women with a history of penicillin allergy, but at low risk for penicillin anaphylaxis, should receive alternative treatment with a cephalosporin such as cefazolin instead of erythromycin or clindamycin. ${ }^{[2]}$ This is due to an increasing resistance of GBS to clindamycin and erythromycin. Reported rates of resistance of GBS to erythromycin range from $25 \%$ to $32 \%$ and resistance to clindamycin from $13 \%$ to $20 \% .{ }^{[2]}$ Vancomycin is an appropriate alternative for patients with a history of anaphylaxis to penicillin and when an isolate is resistant to clindamycin.

An effective GBS vaccine may prevent a broad scope of GBSassociated diseases, such as GBS-EOD, GBS-LOD, spontaneous abortion, stillbirth and maternal bacteraemia. ${ }^{[2,3]}$ One approach of vaccine development is to target the capsular polysaccharide (CPS) of GBS. GBS serological grouping is based on the polysaccharide capsule. It is therefore imperative to determine the GBS serotypes of various geographical regions to develop an inclusive multivalent CPS vaccine.

There are currently 10 serotypes, i.e. Ia, Ib and II - IX. The distribution of the 5 most common GBS serotypes in South Africa (SA) that cause invasive disease is III (55.4\%), Ia (28.2\%), V (7.9\%), II (3.6\%) and Ib (3.4\%), and II (5\%). ${ }^{[4]}$ This is similar to the global distribution. ${ }^{[5]}$ GBS isolates $(7-30 \%)$ are serologically nonserotypeable. ${ }^{[6]}$ In a study conducted at prenatal community clinics in Soweto, SA, researchers looked at the GBS serotype distribution 
rate in colonised mothers, who were swabbed at 5 - 6-week intervals from gestational age 20 - 25 weeks to $37-40$ weeks. The serotype distribution in that study was Ia $(36.2-41.4 \%)$, III (31.3 - 34.9\%), V (10.3 - 15.6\%), II (7.2 - 7.5\%), Ib (3.5 - 4.6\%), IV (2.0 - 4.0\%) and IX $(0.0-3.3 \%) .{ }^{[7]}$ In a recent Canadian study, the most common serotypes seen in maternal colonising GBS strains were III (25\%), Ia (23\%) or V (19\%). ${ }^{[8]}$ The next most common serotypes were II (13\%) and $\mathrm{Ib}(9 \%) .{ }^{[8]}$

The purpose of this laboratory-based observational study was to determine the serotype distribution of rectovaginal colonising isolates from pregnant women in the Pretoria area, SA, and the antimicrobial susceptibility patterns thereof. This serotype distribution, in particular, would be useful to determine the local relevance of a global vaccine, should it be introduced in SA.

\section{Methods}

We examined 69 archived GBS isolates from a study done in 2014, which investigated the prevalence of GBS colonisation in pregnant women between 26 and 37 weeks' gestation. ${ }^{[9]}$ In that study, 284 pregnant women were enrolled from an antenatal clinic and tested for GBS colonisation by Xpert (Cepheid, USA) GBS and culture. The colonisation rate was $25 \%$ by culture and $24 \%$ by Xpert GBS. ${ }^{[7]}$ The GBS isolates from that study were stored in trypticase soy broth with $5 \%$ glycerol.

Microbiology testing for this study was performed at the Tshwane Academic Division, Microbiology Laboratory, National Health Laboratory Service (NHLS). The stored isolates were subcultured on $5 \%$ sheep blood agar and incubated for 24 hours in $5 \% \mathrm{CO}_{2}$. Betahaemolytic colonies were then lawned onto Mueller-Hinton agar, with $5 \%$ sheep blood for minimum inhibitory concentration (MIC) testing using ETEST (bioMérieux, France) strips. Five antibiotics were tested for each isolate, i.e. penicillin, vancomycin, erythromycin, clindamycin and levofloxacin. Plates were incubated for 24 hours in $5 \% \mathrm{CO}_{2}$ at $35-37^{\circ} \mathrm{C}$. The MICs were determined using the latest Clinical and Laboratory Standards Institute (CLSI) breakpoints (2015). The MICs of GBS isolates that tested non-susceptible or resistant for any of the 5 antibiotics were repeated and the results confirmed. Furthermore, 2 observers read the MIC values of all the isolates to minimise inter-observer variability.

As per CLSI guidelines for beta-haemolytic streptococci, macrolidelincosamide-streptogramin B $\left(\mathrm{MLS}_{\mathrm{B}}\right)$ testing was performed on each isolate to test for inducible clindamycin resistance. The isolates were plated on Mueller-Hinton plus 5\% sheep blood agar, after which erythromycin and clindamycin discs were placed next to each other, $12 \mathrm{~mm}$ apart. The plates were incubated at $35-37^{\circ} \mathrm{C}$ in $5 \% \mathrm{CO}_{2}$ for 18 - 24 hours. A 'D-zone' on the side of the clindamycin disc facing the erythromycin disc was taken as positive for the $\mathrm{MLS}_{\mathrm{B}}$ resistance phenotype.

Serotyping of the isolates was conducted at the Respiratory and Meningeal Pathogens Research Unit (RMPRU), University of the
Witwatersrand, Johannesburg, SA. Serotyping was performed using the latex agglutination method, as described by Kwatra et al..$^{[7]}$

\section{Ethical approval}

Ethical approval for the study was obtained from the University of Pretoria Research Ethics Committee (ref. no. 393/2013). All study participants signed informed consent forms on recruitment into the study.

\section{Results}

Antimicrobial susceptibility testing showed that 69 (100\%) isolates were susceptible to penicillin (MIC range $0.032-0.125 \mu \mathrm{g} / \mathrm{mL}$ ) (Table 1). All isolates were susceptible to vancomycin, with 13 (18\%) isolates having an MIC at the breakpoint $(1.0 \mu \mathrm{g} / \mathrm{mL}$ ) (MIC range 0.38 $1.00 \mu \mathrm{g} / \mathrm{mL})$. Sixty (83\%) isolates were sensitive to erythromycin, $8(11 \%)$ were intermediate and $1(1 \%)$ was resistant (MIC range 0.094 - $3.000 \mu \mathrm{g} / \mathrm{mL}$ ). The erythromycin intermediate isolates belonged to serotypes Ia $(n=3)$, III $(n=2)$, IV $(n=1)$ and V $(n=2)$.

Thirty (42\%) isolates were found to be fully susceptible to clindamycin, while $40(56 \%)$ were intermediate and no resistant isolates were detected (MIC range $0.19-0.75 \mu \mathrm{g} / \mathrm{mL}$ ). The clindamycin intermediate isolates belonged to serotypes Ia $(n=23)$, II $(n=1)$, III $(n=8)$, IV $(n=1)$ and V $(n=7)$. Only 3 ( $4 \%$; serotypes Ia, III and $\mathrm{V})$ of our isolates displayed a positive inducible $\mathrm{MLS}_{\mathrm{B}}\left(\mathrm{iMLS}_{\mathrm{B}}\right.$ ) phenotype. All isolates were sensitive to levofloxacin (range 0.38 $1.50 \mu \mathrm{g} / \mathrm{mL}$ ).

The serotype distribution of the 69 isolates was Ia $(54 \% ; n=37)$, III $(20 \% ; n=14), \mathrm{V}(16 \% ; n=11)$, II $(6 \% ; n=4)$, IV $(3 \% ; n=2)$ and Ib $(1 \% ; n=1)$.

\section{Discussion}

The current study characterised the antimicrobial resistance patterns in GBS isolates from pregnant women. In this study, $69(100 \%)$ isolates were fully susceptible to penicillin. In a recent Chinese study on colonising GBS isolates from pregnant women, $100 \%$ of isolates were sensitive to penicillin, ceftriaxone, linezolid and vancomycin. ${ }^{[10]}$ Longtin et al. ${ }^{[11]}$ described a case of GBS with reduced susceptibility to penicillin emerging after long-term suppressive oral penicillin therapy for a prosthetic joint infection.

All isolates in this study were susceptible to vancomycin. There is a paucity of data on vancomycin resistance in GBS isolates, with 2 case reports. These involved 2 patients with invasive GBS infection with significant comorbidities, including diabetes, hypertension, congestive cardiac failure, 1 patient with hypercholesterolaemia and 1 patient with end-stage renal disease, obesity, cor pulmonale and chronic osteomyelitis. ${ }^{[12]}$ Only 1 of these patients had previous prolonged exposure to vancomycin. Both isolates were characterised as belonging to serotype II. In both cases the vancomycin MIC was $4 \mu \mathrm{g} / \mathrm{mL}$.

Macrolides are often regarded as an alternative therapy for penicillin-sensitive patients to treat GBS infections; however,

Table 1. Antimicrobial susceptibility of 69 group B streptococcus isolates to 5 antimicrobial agents

\begin{tabular}{llll}
\hline Antimicrobial agent & $\mathrm{MIC}_{50}, \boldsymbol{\mu g} / \mathrm{mL}$ & $\mathrm{MIC}_{90}, \boldsymbol{\mu g} / \mathrm{mL}$ & Range, $\mu \mathrm{g} / \mathrm{mL}$ \\
\hline Penicillin & 0.047 & 0.064 & $0.032-0.125$ \\
Erythromycin & 0.19 & 0.25 & $0.094-3.000$ \\
Clindamycin & 0.25 & 0.5 & $0.19-0.75$ \\
Vancomycin & 0.75 & 0.5 & $0.38-1.00$ \\
Levofloxacin & 0.5 & 0.75 & $0.38-1.50$
\end{tabular}

MIC $=$ minimum inhibitory concentration 
resistance to macrolides has increased during recent years in several countries, with geographical variations. ${ }^{[13]}$ In the Japanese study by Matsubara et al., ${ }^{[14]}$ researchers found much lower rates of resistance to erythromycin and clindamycin (3\% and $1 \%$, respectively). In a Malaysian study, $23.3 \%$ of isolates were resistant to erythromycin and $17.5 \%$ to clindamycin. ${ }^{[15]}$ The prevalence of resistance among invasive GBS isolates in the USA ranged from $25 \%$ to $32 \%$ for erythromycin and from $13 \%$ to $20 \%$ for clindamycin in reports published during $2006-2009{ }^{[2]}$ Our data suggest a lower level of resistance to these two agents than those observed in the USA and are closer to the Japanese data. ${ }^{[2,14]}$

The CLSI recommends $\mathrm{MLS}_{\mathrm{B}}$ for beta-haemolytic streptococci, which tests for inducible clindamycin resistance. It was found to be the main mechanism of resistance in GBS isolated from the vagina, gastric fluid and ear specimens in a Tunisian study. ${ }^{[13]}$ This phenomenon was noted in only $3(4 \%)$ of our isolates. The serotype distribution of these isolates was Ia, III and V.

All isolates in this study were susceptible to levofloxacin. However, reports of fluoroquinolone-resistant GBS strains have emerged in the past decade, especially in Asia, including China, Japan and Korea. ${ }^{[16]}$ A study by Wu et al ${ }^{[16]}$ confirmed that respiratory samples and elderly patients are two independent risk factors associated with levofloxacin resistance in GBS. The study also found that levofloxacin-resistant GBS isolates belonged mainly to the ST19/serotype III serogroup. ${ }^{[16]}$

Information regarding serotype distribution of GBS strains could guide the development of vaccine candidates. Vaccinating pregnant women against GBS may protect infants from developing invasive GBS disease. Universal screening programmes for maternal GBS colonisation followed by IAP in colonised mothers have been shown to decrease the incidence of EOD. ${ }^{[2]}$ However, it is thought to have a minimal role in the prevention of LOD. ${ }^{[1]}$ GBS maternal vaccination has the potential to decrease EOD, as well as have an impact on LOD. ${ }^{[1]}$

This study showed that serotypes Ia (54\%), III (20\%) and V (16\%) were predominant, which is in accordance with results of other studies conducted among pregnant women in SA. ${ }^{[4]}$ Serotypes Ia and III together accounted for $74 \%$ of the colonised population in our study, while the 3 dominant serotypes accounted for $90 \%$ of all cases. These results are in keeping with those of another SA study that showed that serotype III is the most common cause of EOD, accounting for $41.4 \%$ of all cases, while serotype Ia accounted for $34.7 \%$ of cases. ${ }^{[4]}$ The majority of invasive disease was caused by serotypes Ia, III and $\mathrm{V}^{[4]}$ These 3 serotypes are included in a pentavalent polysaccharide protein conjugate vaccine currently being developed (phase I trial). ${ }^{[17]}$

\section{Study limitations}

The study included isolates from patients seen at a single centre. A further limitation was that we only included rectovaginal colonising isolates and no invasive isolates from neonates. The small sample size is a further limitation of the study.

\section{Conclusions}

GBS isolates remain susceptible to penicillin and vancomycin; however, surveillance for resistance needs to be ongoing. Macrolides should only be used when susceptibility results are available, as significant rates of intermediate resistance have been detected in these isolates. Ninety percent of colonising isolates belong to 3 serotypes, i.e. Ia, III and V. The findings in this study confirm that most of the serotypes in our area will be covered by the current vaccines that are being developed. In view of the small study sample size, we recommend future studies looking into GBS serotype data in SA.

\section{Declaration. None.}

Acknowledgements. Dr Alex Sihlabela, Department of Obstetrics and Gynaecology, Kalafong Hospital, Pretoria, who collected the initial swabs from pregnant women at Kalafong antenatal clinic.

Author contributions. MS: project development, data collection and management, data analysis, manuscript writing; YD: project development, data collection and management, data analysis, manuscript writing; NM: project development, manuscript writing; GK: data collection and management, data analysis, manuscript writing; SM: data collection and management, data analysis, manuscript writing; FI: project development, data collection and management, data analysis, manuscript writing. Funding. Funding for the antimicrobial susceptibility testing was obtained from the National Health Laboratory Service (NHLS) (research grant no. 94443). In addition, funding for the serotyping was obtained from the Respiratory and Meningeal Pathogens Research Unit, University of the Witwatersrand, Johannesburg. These organisations, however, did not have a role in the study design, analysis and interpretation of the data.

Conflicts of interest. Shabir A Madhi received grant funding to support this study from the Department of Science and Technology/National Research Foundation (SA Research Chair Initiative, Chair in Vaccine Preventable Diseases) and the SA Medical Research Council: Respiratory and Meningeal Pathogens Research Unit. His institution also received grants for GBS studies from the Bill and Melinda Gates Foundation, GSK, Minervax and Pfizer.

1. Kobayashi M, Vekemans J, Baker C, Ratner AJ, le Doare K, Schrag SJ. Group B streptococcus vaccine development: Present status and future considerations, with emphasis on perspectives for low and middle income countries. F1000Research 2016;5:2355. https://doi.org/10.12688/f1000research.9363.1

Verani JR, McGee L, Schrag SJ. Prevention of perinatal group B streptococcal disease - revised guidelines from CDC. MMWR Recomm Rep 2019;59(10):1-36.

Johri AK, Paoletti LC, Glaser P, et al. Group B streptococcus: Global incidence and vaccine development. Nat Rev Microbiol 2006;4(12):932-942. https://doi.org/10.1038/nrmicro1552 4. Dangor Z, Cutland C, Izu A, et al. Temporal changes in invasive group B streptococcus serotypes:
Implications for vaccine development. PLoS ONE 2016;11(12):e0169101. https://doi.org/10.1371/ journal.pone. 0169101

5. Madrid L, Seale A, Kohli-Lynch M, et al. Infant group B streptococcal disease incidence and serotypes worldwide: Systematic review and meta-analyses. Clin Infect Dis 2017;65(S2):S160-S172. https://doi. org/10.1093/cid/cix656

6. Dangor Z, Lala S, Cutland C, et al. Burden of invasive group B streptococcus disease and early neurological sequelae in South African infants. PLoS ONE 2015;10(4):e0123014. https://doi org/10.1371/journal.pone.0123014

7. Kwatra G, Adrian P, Shiri T, et al. Serotype-specific acquisition and loss of group B streptococcus rectovaginal colonization in late pregnancy. PLoS ONE 2015;9(6):e98778. https://doi.org/10.1371/journal pone. 0098778

8. Teatero S, Ferrieri P, Martin I, et al. Serotype distribution, population structure and antimicrobial resistance of group B streptococcus strains recovered from colonized pregnant women. J Clin Micro 2017;55(2):412-422. https://doi.org/10.1128/JCM.01615-16

9. Said M, Dangor Y, Mbelle N, et al. Comparison of Xpert GBS v. culture for rapid detection of group

B streptococcus in pregnant women: Sensitivity, specificity and predictive values. S Afr Med J B streptococcus in pregnant women: Sensitivity, specificity and predictive
2018;108(12):1032-1035. https://doi.org/10.7196/SAMJ.2018.v108i12.13079

10. Ji W, Zhang L, Guo Z, et al. Colonization prevalence and antibiotic susceptibility of group B streptococcus in pregnant women over a 6-year period in Dongguan, China. PLoS ONE 2017;12(8):e018083. https://doi.org/10.1371/journal.pone.0183083

11. Longtin J, Vermeiren C, Shahinas D, et al. Novel mutations in a patient isolate of Streptococcus agalactiae with reduced penicillin susceptibility emerging after long term oral suppressive therapy Antimicrob Agents Chemother 2011;55(6):2983-2985. https://doi.org/10.1128/AAC.01243-10

12. Park C, Nichols M, Schrag S. Two cases of invasive vancomycin resistant group B streptococcus infection. N Engl J Med 2014;370(9):885-886. https://doi.org/10.1056/NEJMc1308504

13. Hraoui M, Boutiba-Ben Boubaker I, Rachdi M, et al. Macrolide and tetracycline resistance in clinical strains of Streptococcus agalactiae isolated in Tunisia. J Med Microbiol 2012;61:1109-1113. https://doi org/10.1099/jmm.0.037853-0

14. Matsubara K, Nishiyama Y, Katayama K, et al. Change of antimicrobial susceptibility of group B streptococci over 15 years in Japan. J Antimicrob Chemother 2001;48:579-582. https://doi.
org/10.1093/jac/48.4.579

15. Eskandarian N, Ismail Z, Neela V, et al. Antimicrobial susceptibility profiles, serotype distribution and virulence determinants among invasive, non-invasive and colonising Streptococcus agalactiae (group
vition B streptococcus) from Malaysian patients. Eur J Clin Microbiol Infect Dis 2015;34(4):579-584. https:// B streptococcus) from Malaysian patient

doi.org/10.1007/s10096-014-2265-x
16. Wu C, Lai J-F, Huang I-W, et al. Multic

16. Wu C, Lai J-F, Huang I-W, et al. Multiclonal emergence of levofloxacin-resistant group B streptococcus, Taiwan. J Antimicrob Chemother 2017;72(12):3263-3271. https://doi.org/10.1093/jac/dkx297

17. Lin SM, Zhi Y, Ahn KB, et al. Status of group B streptococcal vaccine development. Clin Exp Vaccine Res 2018;7(1):76-81. https://doi.org/10.7774/cevr.2018.7.1.76

Accepted 27 May 2020 